patienten erhielten auch 75 mg Clopidogrel täglich.

Unter anderem Patienten mit Asthma, einem Quincke-Ödem oder einer sich entwickelnden Allergie durften an der Studie nicht teilnehmen. Geprüft wurde nach der Desensibilisierung die Aktivität von ASS gegen die Blutplättchen mithilfe der Lichttransmissionsaggregometrie (LTA), bei der die Veränderung der Transmission langwelligen Lichts gemessen wird. Die Thrombozytenaggregation wurde in dem Testsystem unter Stimulation mit Arachidonsäure $\leq 20 \%$ bestimmt. Die Messung erfolgte an Tag 1 sowie nach sechs bis acht Wochen.
Die Desensibilisierung verlief problemlos. Bei 24 Patienten war eine Angioplastie vorgenommen worden, 14 Patienten erhielten einen Medikamente freisetzenden Koronarstent. Innerhalb des Beobachtungszeitraums von sechs bis acht Wochen sei es zu keinem größeren kardialen Ereignis gekommen, so die Kardiologen. Stentthrombosen wurden nicht beobachtet. Nach diesem Zeitraum seien keine Hypersensitivitätssymptome als Reaktion auf ASS aufgetreten. Am Tag 1 nach der Desensibilisierung war die Thrombozytenaggregationshemmung gemessen anhand der LTA - bei $86 \%$ der Patienten mit ASS-Hypersensitivität er- folgreich (25 von 29 Patienten). Nach sechs bis acht Wochen lag der Anteil bei $95 \%$ (20 von 21 Patienten), in der Kontrollgruppe bei $100 \%$.

Fazit: Bei Patienten mit ASS-Hypersensitivität lässt sich mithilfe einer kurzzeitigen oralen Desensibilisierung mit dem Cyclooxygenasehemmer wieder eine erfolgreiche Thrombozytenaggregationshemmung erzielen.

Peter Leiner

Manzo-Silberman S et al. Effect of Rapid Desensitization on Platelet Inhibition and Basophil Activation in Patients with Aspirin Hypersensitivity and Coronary Disease. Eur Heart J Cardiovasc Pharmacother 2016; pii: pvw018

\title{
Luftreiniger reduziert Pollenkonzentration in geschlossenen Räumen
}

\author{
Pollenbelastung macht Allergikern nicht nur im Freien, sondern auch in \\ Wohn- und Arbeitszimmern zu schaffen. Berliner Allergologen haben \\ untersucht, ob Geräte zur Luftreinigung hier weiterhelfen können.
}

Eine Arbeitsgruppe des Allergiezent-
rums der Berliner Charité um Prof.
Dr. Karl-Christian Bergmann hat junge
erwachsene Patienten mit pollenallergi-
scher Rhinitis und/oder Konjunktivitis
für einen dreistufigen Test in eine Pol-
lenexpositionskammer gesetzt. Dabei
lief zunächst eine halbe Stunde lang in
einem Abstand von $150 \mathrm{~cm}$ ein Luftrei-
niger der Marke Philips AC4012, um he-
rauszufinden, ob das Gerät selbst das
Beschwerdebild der Probanden beein-
flusst. Auf diese erste folgte eine zweite Phase, in der die Versuchspersonen $90 \mathrm{Mi}-$ nuten lang einer Konzentration von 4.000 Graspollen je Kubikmeter ausgesetzt wurden. Der Luftreiniger war zwar eingeschaltet, der Filtereinsatz aber ausgebaut. Während der dritten Phase lief der Luftreiniger voll ausgerüstet mit einem Filter bei gleicher Pollenkonzentration über einen Zeitraum von ebenfalls 90 Minuten.

Gemessen wurden unter anderem die Symptomenscores bezogen auf die Augen (Jucken, Reizung, Rötung, Tränen), die Nase (Jucken, Niesen, Triefen, Verstopfung) und die Bronchien (Giemen,
Husten, Kurzatmigkeit, Asthma). Für jedes Symptom gab es 0 (keine Beschwerden) bis 3 Punkte (heftige Beschwerden), sodass je untersuchtes Organ im schlechtesten Fall 12 Punkte zu Buche kamen.

In Phase 1 traten kaum Beschwerden auf. Anders in Phase 2: Hier lagen die Symptomenscores zwischen 4 und 6. In Phase 3, also bei Pollenbelastung mit laufendem und filterbewehrtem Luftreiniger, gingen die Symptomenwerte im Mittel wieder auf unter 1 Punkt zurück, und zwar sowohl im Durchschnitt für alle untersuchten Organe als auch speziell für den Nasenscore.

Obwohl eine Pollenallergie in erster Linie als ein Phänomen angesehen wird, das sich durch Belastung an der frischen Luft manifestiert, haben Studien gezeigt, dass die Pollen von Gräsern und Birken auch in Innenräumen nachzuweisen sind. Dort können sie empfindlichen Patienten noch lange nach der eigentlichen Pollensaison allergische Beschwerden verursachen. Folgt man den Ergebnissen der vorliegenden Untersuchung, können Luftreiniger diese Beschwerden lindern.
„Unser Studienprotokoll ist geeignet, die Wirksamkeit von Luftreinigern zu untersuchen, und der getestete Reiniger hat die von Graspollen verursachten klinischen Symptome in einem Innenraum wirksam reduziert", schreiben die Autoren. Unterstützt hat die Studie die Firma Philips, die das verwendete Gerät herstellt. Dennoch waren, wie die Forscher einräumen, die finanziellen Ressourcen begrenzt. Das Team musste sich deshalb mit vier Probanden bescheiden.

Dr. Robert Bublak

Bergmann KC et al. A novel experimental technology for testing efficacy of air purifiers on pollen reduction. Allergo J Int 2017;26:1-6

\section{Kommentar}

Allergenkarenz fällt vor allem Pollenallergikern naturgemäß schwer. Dieser Studie zufolge kann ein Luftreiniger die Allergenbelastung in den eigenen vier Wänden spürbar reduzieren. Bevor wir unseren Patienten nun die Anschaffung eines entsprechenden Geräts empfehlen, gilt es weitere, ausführlichere Studien abzuwarten. Die Lektüre dieser Arbeit im Original lohnt sich aber bereits jetzt.

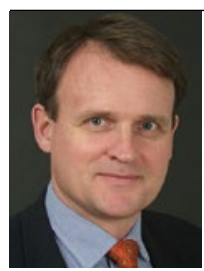

Prof. Dr. Thilo Jakob Direktor der Klinik für Dermatologie und Allergologie Universitätsklinikum Gießen u. Marburg, Standort Gießen 